\title{
The role of scheduled second TACE in early-stage hepatocellular carcinoma with complete response to initial TACE
}

\author{
Jung Hee Kim ${ }^{1 *}$, Dong Hyun Sinn ${ }^{1 *}$, Sung Wook Shin ${ }^{2}$, Sung Ki Cho², Wonseok Kang ${ }^{1}$, Geum-Youn Gwak', \\ Yong-Han Paik', Joon Hyeok Lee', Kwang Cheol Koh', Seung Woon Paik', and Moon Seok Choi ${ }^{1}$ \\ Departments of ${ }^{1}$ Medicine and ${ }^{2}$ Radiology, Samsung Medical Center, Sungkyunkwan University School of Medicine, Seoul, Korea
}

Background/Aims: We investigated the outcomes of early-stage hepatocellular carcinoma (HCC) patients who showed a complete response (CR) to initial transarterial chemoembolization (TACE), with a focus on the role of scheduled TACE repetition.

Methods: A total of 178 patients with early-stage HCC who were initially treated with TACE and showed a CR based on the modified Response Evaluation Criteria in Solid Tumors (mRECIST) criteria on one month follow-up computed tomography (CT) were analyzed. Among them, 90 patients underwent scheduled repetition of TACE in the absence of viable tumor on CT.

Results: During a median follow-up period of 4.6 years (range: $0.4-8.8$ years), mortality was observed in 71 patients (39.9\%). The overall recurrence-free and local recurrence-free survival rates at 1 year were $44.4 \%$ and $56.2 \%$. In the multivariable model, scheduled repetition of TACE was an independent factor associated with survival (hazard ratio [95\% confidence interval]: 0.56 [0.34-0.93], $P=0.025$ ). When stratified using Barcelona clinic liver cancer (BCLC) stage, scheduled repetition of TACE was associated with a favorable survival rate in BCLC stage $A$ patients, but not in BCLC 0 patients.

Conclusions: Scheduled repetition of TACE was associated with better survival for early-stage HCC patients showing a CR after initial TACE, especially in BCLC stage A patients. (Clin Mol Hepatol 2017;23:42-50)

Keywords: Carcinoma, Hepatocellular; Transarterial chemoembolization; Survival

\section{INTRODUCTION}

Transarterial chemoembolization (TACE) is the most commonly used nonsurgical treatment modality for patients with hepatocellular carcinoma (HCC).' Tumor necrosis can be achieved by the combined effects of antitumor chemotherapy and selective ischemia of tumor tissue. ${ }^{1}$ TACE has shown survival benefit over best supportive care for patients with unresectable HCC in two randomized controlled trials. ${ }^{2,3}$ It is the first line treatment recommended for Barcelona clinic liver cancer (BCLC) stage B (multinodular, preserved liver function, and good performance status) HCC. ${ }^{4,5}$ For early stage HCC (BCLC stage 0 or A), resection or local ablation is the first line recommended therapy. ${ }^{1,4,5}$ However, sometimes, TACE is the only option for early stage HCC, as resection or

\section{Abbreviations:}

AFP, alpha fetoprotein; BCLC, Barcelona clinic liver cancer; HCC, hepatocellular carcinoma; mRECIST, modified response evaluation criteria in solid tumors; TACE, transarterial chemoembolization

\section{Corresponding author : Moon Seok Choi}

Department of Medicine, Samsung Medical Center, Sungkyunkwan University School of Medicine, 81 Irwon-ro, Gangnam-gu, Seoul 06351, Korea

Tel: +82-2-3410-3409, Fax: +82-2-3410-6983

E-mail:drmschoi@gmail.com 
local ablation is infeasible due to poor hepatic reservoir function and/or improper tumor location for some cases. Indeed, the global HCC BRIDGE study, a multiregional large-scale longitudinal cohort study including 18,031 patients from 14 countries, has shown that TACE is a widely practice for HCC across the BCLC stages, including early stage $\mathrm{HCC}^{6}$

Once TACE is performed, its effect is usually evaluated by modified Response Evaluation Criteria in Solid Tumors (mRECIST) criteria, ${ }^{7}$ based on tumor viability defined by the uptake of contrast agent in the arterial phase of dynamic imaging studies. When viable tumor is noticed, repetition of TACE is considered. However, it is well known that complete response by radiological evaluation does not always correspond to total necrosis of the tumor by histological evaluation. ${ }^{8,9}$ Hence, scheduled repetition of TACE (second TACE) for early stage tumor with complete response by radiological evaluation after the first TACE has been performed in some centers based on physician's preference without significant data to support this approach. To the best of our knowledge, no study has evaluated whether scheduled second TACE for those who showed complete response on 1 month follow-up computed tomography (CT) is associated with better outcome than on-demand approach. The objective of this study was to determine the long-term outcome of early stage HCC patients who showed complete response after the first TACE, with special focus on the role of scheduled repetition treatment strategy.

\section{MATERIALS AND METHODS}

\section{Study population}

This is a retrospective cohort study. We screened Samsung Medical Center HCC registry for the period from January 2007 to December 2012. Detailed description of Samsung Medical Center HCC registry has been described in our previous paper..$^{10}$ Briefly, all newly-diagnosed HCC patients who received care at our institution were prospectively registered. HCC was diagnosed either histologically or clinically according to the regional guideline." Well-trained abstractors collected data of HCC patients including age at diagnosis, gender, date of diagnosis, etiology, liver function, tumor characteristics, tumor stage, and initial treatment modality. From this registry, we screened a total of 577 patients diagnosed at early-stage (defined as the BCLC stage 0 or A evaluated with $\mathrm{CT}$ or magnetic resonance imaging [MRI]) who received conventional lipiodol TACE as an initial treatment. Among them, we enrolled 178 patients who showed complete response by mRECIST criteria at 1-month follow-up CT evaluation after the first TACE. This study was reviewed and approved by the Institutional Review Board at Samsung Medical Center. Because the study is based on the retrospective analysis of existing administrative and clinical data, the requirement of obtaining informed patient consent was waived by the Institutional Review Board.

\section{Primary end-point, exposure, and other variables}

The primary end-point was overall survival, defined as the time from the initial diagnosis of HCC to death. All patients were followed-up from the baseline to September 2015. Patient survival data were collected from National Statistics Service. Therefore, all deaths at the time of survival assessment were certified. The exposure was scheduled second TACE in the absence of radiological evidence of viable HCC after the first TACE. These patients were defined as scheduled second TACE group. Otherwise, patients were categorized as on-demand group, if they received second treatment when tumor became apparent by radiological evaluation. We also collected variables known to affect patient prognosis, such as age at diagnosis, gender, etiology of liver disease, serum alpha-fetoprotein levels, Child-Pugh score, maximal tumor size, and tumor number. Tumor recurrence was reviewed and categorized into local recurrence, the recurrence of the initial TACE target lesion or remote new recurrence, the recurrence of other site except the initial lesion.

\section{TACE procedure and follow-up}

TACE was performed by six experienced intervention radiologists who had 3 to 16 years of experiences in TACE. After selective arteriography of the superior mesenteric, celiac, and common hepatic arteries using a 5-French catheter, the hepatic artery was catheterized with a coaxial microcatheter. After the microcatheter was positioned into or as close as possible to the tumor feeding branch, an emulsion of doxorubicin hydrochloride (adriamycin; Idong, Seoul, Korea) and iodized oil (Lipiodol; Guerbet, Aulnaysous-Bois, France) was slowly infused through the catheter. Oily TACE was performed as selectively as possible and a microcatheter was routinely used. The doses of iodized oil and doxorubicin were determined based on the size and vascularity of the tumor; the maximum doses of iodized oil and doxorubicin for a single session of TACE being $25 \mathrm{~mL} \mathrm{mg}$ and $70 \mathrm{mg}$, respectively. Infusion of the lipiodol mixture was followed by particulate embolization 
with 1- to 2-mm-diameter gelatin sponge pledgets (Cutanplast; MasciaBrunelli, Milan, Italy). After TACE, patients were followed up one month later with dyanamic $\mathrm{CT}$ to evaluate tumor response. Those who received scheduled second TACE received the second TACE in similar manner, and underwent CT scan at one month later. Afterward, patients were monitored at 3-6 months interval with CT or MRI. Those in the on-demand group had the same follow-up protocols, except that they did not received the scheduled second TACE.

\section{Statistical analyses}

Baseline characteristics between those who received scheduled second TACE and on-demand approach were compared using chisquare test, Fisher's exact test, $t$-test or Mann-Whitney test as appropriate. The overall survival rate was estimated using KaplanMeier method and compared using log-rank test. Cox-proportional hazard model was conducted to see whether scheduled second TACE is associated with overall survival, recurrence-free survival, and local tumor progression-free survival. Multivariable model was based on factors that showed significant association at unadjusted analysis $(P<0.05)$. Subgroup analysis was performed to de- termine if any subgroup had survival advantage. A two-sided $p$ value of less than 0.05 was considered as statistically significant. IBM SPSS V23.0 (SPSS Inc., IBM Corporation, Chicago, IL, USA) software was used for all statistical analyses.

\section{RESULTS}

\section{Overall survival and recurrence-free survival}

The baseline characteristics of patients are shown in Table 1 and Supplementary Table 1. Their mean age was 64.3 years and male comprised $69.7 \%$. Hepatitis B virus was a major etiology. BCLC stage was 0 in 51 (28.7\%) patients. During a median 4.6 years of follow-up (range: $0.4-8.8$ years), mortality was observed in $71(39.9 \%)$ patients. The overall survival rates at 1,3 , and 5 years were $93.3 \%, 86.5 \%$, and $60.4 \%$, respectively (Fig. 1A). During the follow-up, recurrence was observed in 135 (75.8\%) patients. The recurrence-free survival rates at 1, 2, and 3 years were of $44.4 \%$, $18.5 \%$ and $12.6 \%$, respectively (Fig. 1B). Local recurrence was noticed in $103(57.9 \%)$ patients. Local tumor recurrence-free survival

Table 1. Comparison of baseline characteristics

\begin{tabular}{|c|c|c|c|c|}
\hline Characteristics & All $(n=178)$ & On-demand group $(n=90)$ & Scheduled second TACE $(n=88)$ & $P$-value \\
\hline Age (years) & $64.3 \pm 10.5$ & $64.5 \pm 10.9$ & $64.1 \pm 10.0$ & 0.81 \\
\hline Male & $124(69.7)$ & $61(67.8)$ & $63(71.6)$ & 0.35 \\
\hline Etiology (HBV) & $123(69.1)$ & $60(66.7)$ & $63(71.6)$ & 0.29 \\
\hline Child-Pugh score & & & & 0.12 \\
\hline A & $146(82.0)$ & $77(85.6)$ & $69(78.5)$ & \\
\hline B & $32(18.0)$ & $13(14.4)$ & $19(21.5)$ & \\
\hline MELD score & $6.4(4.1-8.6)$ & $6.4(3.8-8.8)$ & $6.3(4.2-8.5)$ & 0.47 \\
\hline Tumor number & & & & 0.25 \\
\hline Single & $117(65.7)$ & $60(66.7)$ & $57(64.8)$ & \\
\hline Two-three & $61(34.3)$ & $30(33.3)$ & $31(35.2)$ & \\
\hline Tumor size $(\mathrm{cm})$ & $2.0(1.3-2.6)$ & $1.7(1.3-2.5)$ & $2.0(1.5-3.0)$ & 0.07 \\
\hline$\leq 2.0^{*}$ & 104 (58.4) & $58(64.4)$ & $46(52.3)$ & \\
\hline$>2.0^{\dagger}$ & 74 (41.6) & 32 (35.6) & $42(47.7)$ & \\
\hline BCLC stage & & & & 0.001 \\
\hline 0 & $51(28.7)$ & $36(40.0)$ & $15(17.0)$ & \\
\hline A & $127(71.3)$ & $54(60.0)$ & $73(83.0)$ & \\
\hline $\operatorname{AFP}(\mathrm{ng} / \mathrm{mL})$ & $20.1(6.8-94.6)$ & $19.9(6.7-72.3)$ & $20.1(8.0-97.3)$ & 0.36 \\
\hline
\end{tabular}

Values are presented as mean \pm standard deviation, median (quartile) or $n(\%)$.

TACE, transarterial chemoembolization; HBV, hepatitis B virus; MELD, model for end stage liver disease; BCLC stage, Barcelona clinic liver cancer stage; AFP, alpha fetoprotein.

"The median tumor size was $1.5 \mathrm{~cm}$ (range: 1.0-2.0 cm); ${ }^{\dagger}$ The median tumor size was $2.8 \mathrm{~cm}$ (range: 2.1-6.0 cm). 

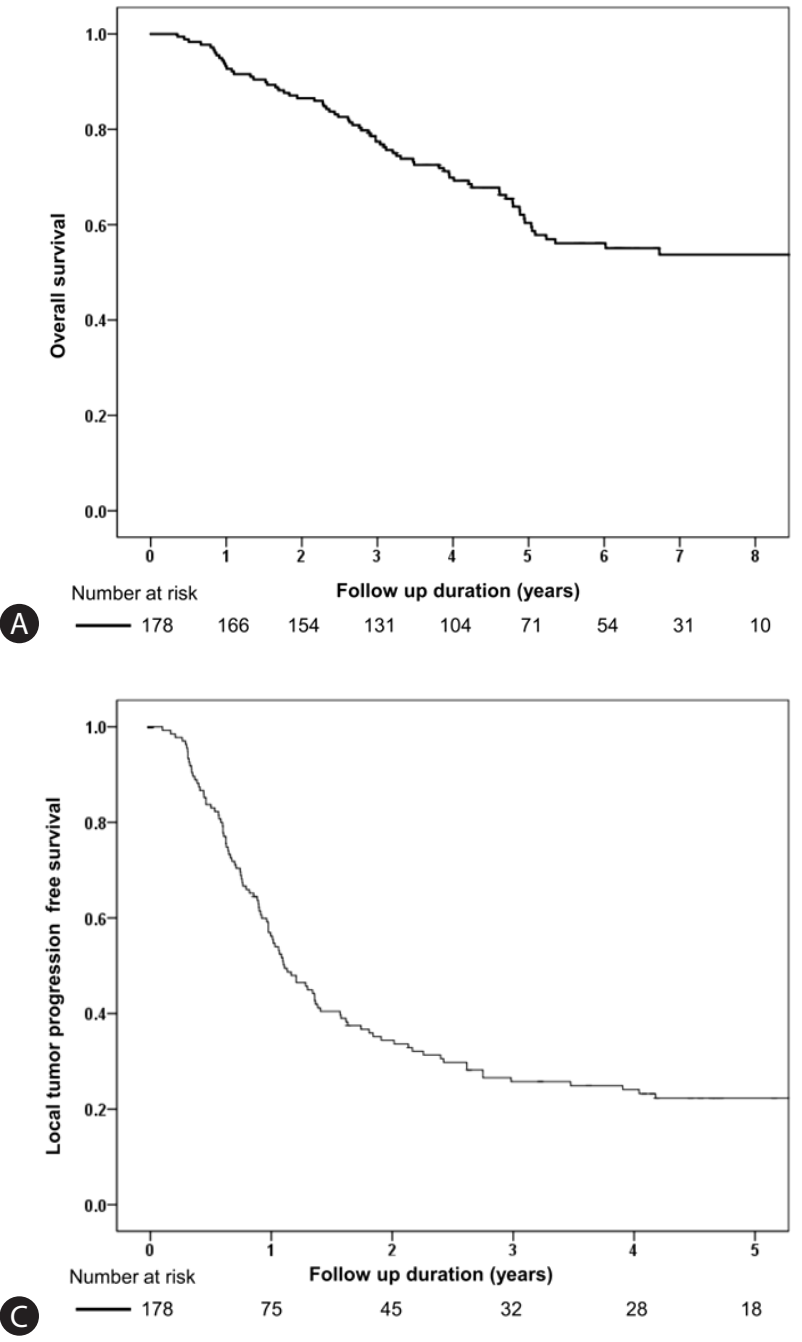

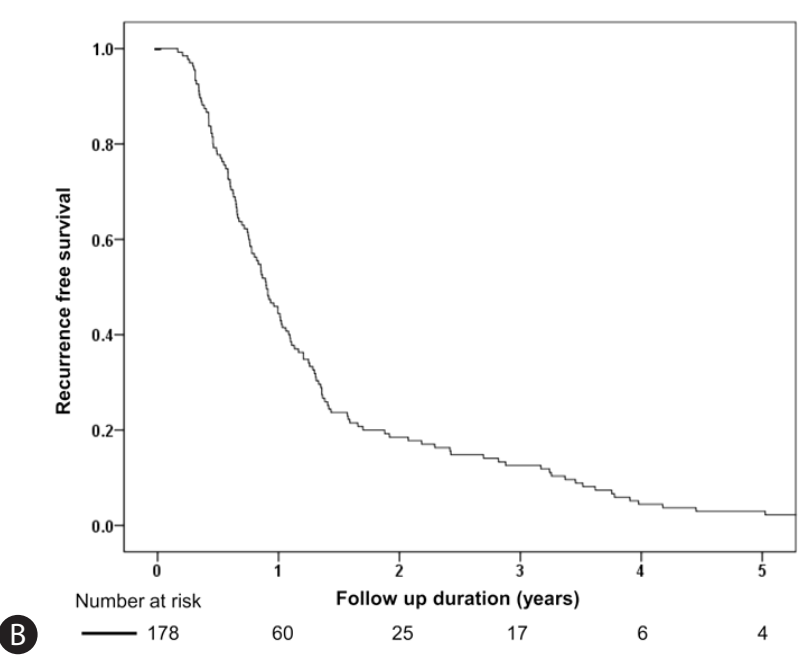

Figure 1. Prognosis of very early- or early-stage hepatocellular carcinoma patients treated with TACE. (A) Overall survival, (B) Recurrence-free survival and (C) Local tumor recurrence-free survival. TACE, transarterial chemoembolization.

Table 2. Prognostic factors for overall survival

\begin{tabular}{|c|c|c|c|c|}
\hline Characteristics & Unadjusted HR (95\% Cl) & $P$-value & Multivariable HR $(95 \%$ CI) & $P$-value \\
\hline Age (year) & $1.03(1.01-1.06)$ & 0.006 & $1.02(0.99-1.04)$ & 0.070 \\
\hline Male & $1.05(0.63-1.76)$ & 0.82 & & \\
\hline Etiology (HBV vs. other) & $0.47(0.29-0.75)$ & 0.002 & $0.50(0.30-0.83)$ & 0.008 \\
\hline Child-Pugh class B (vs. A) & $2.19(1.27-3.75)$ & 0.004 & $1.88(1.06-3.34)$ & 0.029 \\
\hline AFP (log ng/mL) & $0.99(0.86-1.14)$ & 0.95 & & \\
\hline BCLC stage A (vs. 0) & $2.86(1.50-5.45)$ & 0.001 & $3.07(1.54-6.12)$ & 0.001 \\
\hline Tumor recurrence & $0.97(0.59-1.58)$ & 0.89 & & \\
\hline \multicolumn{5}{|l|}{ Treatment strategy } \\
\hline On-demand group & Reference & & Reference & \\
\hline Scheduled second TACE & $0.79(0.49-1.26)$ & 0.33 & $0.56(0.34-0.93)$ & 0.025 \\
\hline
\end{tabular}

$\mathrm{HR}$, harzard ratio; $\mathrm{Cl}$, confidence interval; HBV, hepatitis B virus; AFP, alpha fetoprotein; BCLC stage, Barcelona clinic liver cancer stage; TACE, transarterial chemoembolization. 
Table 3. Prognostic factors for recurrence-free survival

\begin{tabular}{|c|c|c|c|c|}
\hline Characteristics & Univariate HR (95\% Cl) & $P$-value & Multivariable HR (95\% Cl) & $P$-value \\
\hline Age (year) & $1.01(0.99-1.03)$ & 0.43 & & \\
\hline Male & $1.22(0.85-1.76)$ & 0.28 & & \\
\hline Etiology (HBV vs. other) & $1.04(0.71-1.54)$ & 0.83 & & \\
\hline Child-Pugh class B (vs. A) & $1.82(1.10-3.01)$ & 0.020 & $1.29(0.77-2.18)$ & 0.32 \\
\hline AFP (log ng/mL) & $1.02(0.92-1.13)$ & 0.75 & & \\
\hline BCLC stage A (vs. 0) & $2.58(1.71-3.89)$ & $<0.001$ & $2.46(1.60-3.78)$ & $<0.001$ \\
\hline \multicolumn{5}{|l|}{ Treatment strategy } \\
\hline On demand group & Reference & & Reference & \\
\hline Scheduled second TACE & $1.21(0.86-1.17)$ & 0.27 & $1.01(0.71-1.43)$ & 0.96 \\
\hline
\end{tabular}

HR, harzard ratio; $\mathrm{Cl}$, confidence interval; HBV, hepatitis B virus; AFP, alpha fetoprotein; BCLC stage, Barcelona clinic liver cancer stage; TACE, transarterial chemoembolization.

Table 4. Prognostic factors for local tumor recurrence-free survival

\begin{tabular}{|c|c|c|c|c|}
\hline Characteristics & Univariate $\mathrm{HR}(95 \% \mathrm{Cl})$ & $P$-value & Multivariable HR (95\% Cl) & $P$-value \\
\hline Age (year) & $0.99(0.97-1.01)$ & 0.23 & & \\
\hline Male & $1.05(0.69-1.60)$ & 0.83 & & \\
\hline Etiology (HBV vs. other) & $1.26(0.81-1.98)$ & 0.31 & & \\
\hline Child-Pugh class B (vs. A) & $1.48(0.86-2.56)$ & 0.16 & & \\
\hline AFP (log ng/mL) & $1.05(0.93-1.17)$ & 0.44 & & \\
\hline BCLC stage A (vs. 0) & $1.99(1.27-3.10)$ & 0.002 & $2.23(1.40-3.57)$ & 0.001 \\
\hline \multicolumn{5}{|l|}{ Treatment strategy } \\
\hline On-demand group & Reference & & Reference & \\
\hline Scheduled second TACE & $0.94(0.64-1.38)$ & 0.74 & $0.72(0.48-1.09)$ & 0.12 \\
\hline
\end{tabular}

$\mathrm{HR}$, harzard ratio; $\mathrm{Cl}$, confidence interval; HBV, hepatitis B virus; AFP, alpha fetoprotein; BCLC stage, Barcelona clinic liver cancer stage; TACE, transarterial chemoembolization.

rate at 1,2 , and 3 years were $56.2 \%, 34.4 \%$ and $25.8 \%$, respectively (Fig. 1C).

\section{Factors associated with overall, recurrence-free, and local recurrence-free survival}

Age, etiology, Child-Pugh class, and BCLC stage were factors associated with overall survival in unadjusted analysis (Table 2). The 5-year survival rate in patients who received scheduled second TACE was higher than that in the on-demand group, although the difference was not statistically significant (66.3\% vs. $54.2 \%$, $P=0.33)$. However, when baseline characteristics were compared, those with scheduled second TACE had more advanced BCLC stage than those in the on-demand group (Table 1). In a multivariable model, scheduled second TACE and etiology of hepatitis $B$ virus infection (vs. others) were independent good prognostic factors while Child-Pugh class B (vs. A) and BCLC stage A (vs. 0) were independent poor prognostic factors associated with the overall survival (Table 2). Child-Pugh class and BCLC stage were associated with recurrence-free survival in unadjusted analysis. BCLC stage A (vs. 0) was poor prognostic factors associated with recurrence-free survival (Table 3) and local recurrence-free survival in multivariable analysis (Table 4).

Treatment strategy (scheduled second TACE vs. on-demand group) was not associated with recurrence-free survival (39.7\% vs. $49.2 \%$ at 1 year, $P=0.26)$ and local recurrence-free survival (68.3\% vs. $66.8 \%$ at 1 year, $P=0.38)$.

\section{Long-term outcome of scheduled second TACE or on-demand according to $\mathrm{BCLC}$ stage}

Sub-group analysis according to BCLC stage was performed. The baseline characteristics of BCLC stage 0 and $A$ are shown in Supplementary Table 1. In BCLC 0 patients, there were no signifi- 
Table 5. Local recurrence overall recurrence and survival after scheduled second TACE stratified by BCLC stage

\begin{tabular}{|c|c|c|c|c|c|c|c|c|c|}
\hline Subgroup & $\begin{array}{l}\text { Local } \\
\text { recurrence free } \\
\text { survival rate }(\%) \\
\text { at } 1 \text { year }\end{array}$ & $\begin{array}{l}\text { Adjusted }^{*} \\
\text { HR }(95 \% \mathrm{CI})\end{array}$ & $P$-value & $\begin{array}{c}\text { Overall } \\
\text { recurrence free } \\
\text { survival rate (\%) } \\
\text { at } 1 \text { years }\end{array}$ & $\begin{array}{l}\text { Adjusted } \\
\text { HR }(95 \% \mathrm{Cl})\end{array}$ & $P$-value & $\begin{array}{c}\text { Overall } \\
\text { survival rate } \\
(\%) \text { at } 5 \text { years }\end{array}$ & $\begin{array}{c}\text { Adjusted } \\
\text { HR }(95 \% \mathrm{Cl})\end{array}$ & $P$-value \\
\hline $\begin{array}{c}\mathrm{BCLC} 0 \\
(\mathrm{n}=51)\end{array}$ & 69.0 vs. 84.6 & $\begin{array}{c}1.48 \\
(0.68-3.20)\end{array}$ & 0.31 & 58.6 vs. 42.1 & $\begin{array}{c}0.90 \\
(0.46-1.77)\end{array}$ & 0.77 & 76.0 vs. 85.6 & $\begin{array}{c}0.82 \\
(0.21-3.11)\end{array}$ & 0.77 \\
\hline $\begin{array}{l}\text { BCLC A } \\
(n=127)\end{array}$ & 44.7 vs. 50.6 & $\begin{array}{c}0.60 \\
(0.38-0.95)\end{array}$ & 0.029 & 42.1 vs. 32.7 & $\begin{array}{c}1.04 \\
(0.68-1.59)\end{array}$ & 0.84 & 39.1 vs. 62.1 & $\begin{array}{c}0.58 \\
(0.34-0.98)\end{array}$ & 0.034 \\
\hline
\end{tabular}

TACE, transarterial chemoembolization; BCLC, Barcelona clinic liver cancer; $\mathrm{HR}$, hazard ratio; $\mathrm{Cl}$, confidence interval.

*Adjusted for tumor number, size and Child-Pugh score.
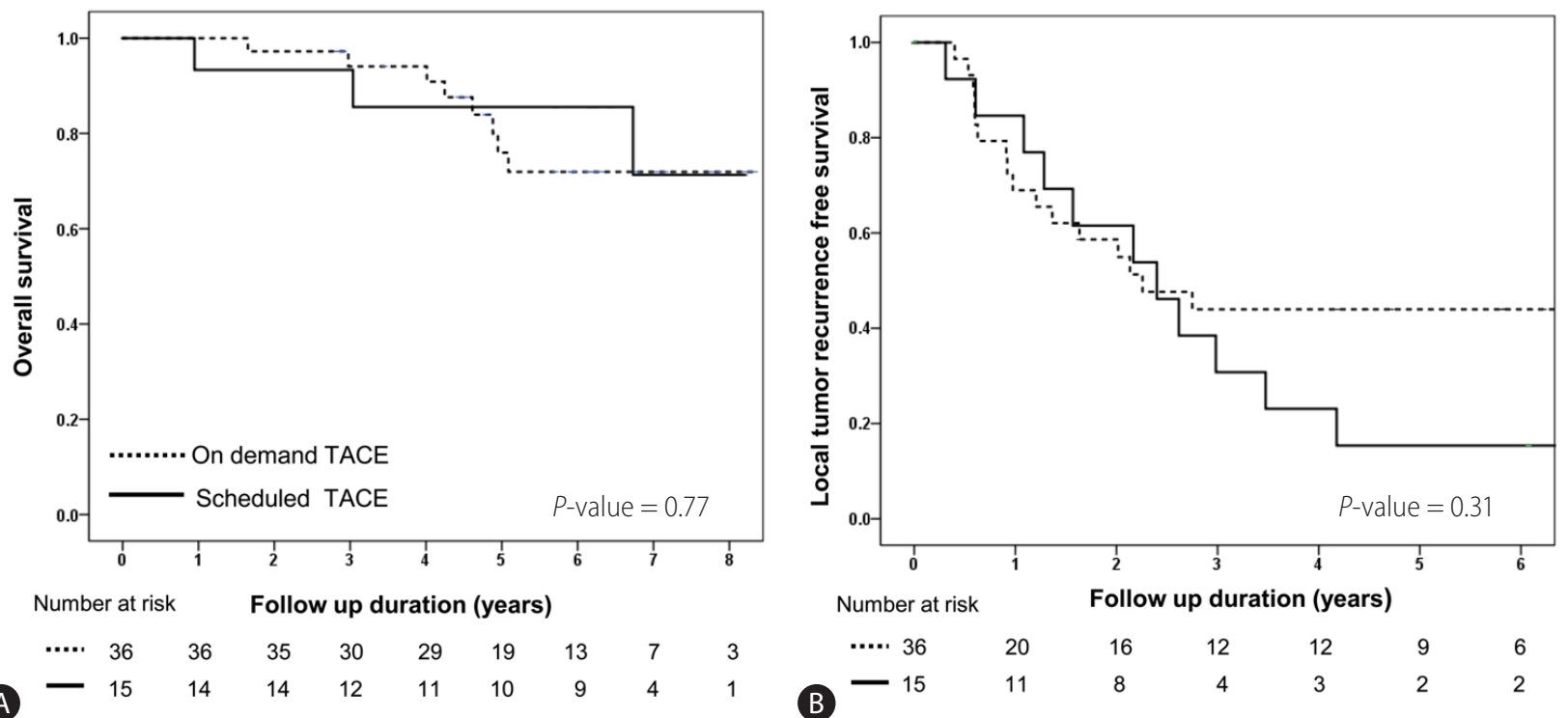

Figure 2. Comparison of overall survival and local tumor recurrence-free survival in scheduled second TACE and on-demand TACE groups of BCLC O stage patients $(n=51)$. Kaplan-Meier curves showing the overall survival rate $(A)$ and local tumor recurrence-free survival $(B)$ in the scheduled second TACE $(n=15)$ and on-demand groups $(n=36)$. There was no significant differences in overall survival $(76.0 \% \mathrm{vs}$. $85.6 \%$ at 5 years, $P=0.77)$ or local tumor recurrence-free survival $(69.0 \%$ vs. $84.6 \%$ at 1 year, $P=0.31$ ) between the second TACE and on-demand groups. TACE, transarterial chemoembolization; BCLC, Barcelona clinic liver cancer.

cant $(P>0.05)$ differences in overall, recurrence, and local-recurrence free survival between patients who received scheduled second TACE and those who received on-demand therapy (Table 5, Fig. 2). However, in BCLC stage A patients, the overall survival and local recurrence-free survival were significantly higher in those who received scheduled second TACE (Fig. 3). There was no difference in overall recurrence free survival by treatment strategy in BCLC A patients (Table 5).

\section{DISCUSSION}

For very early or early stage $\mathrm{HCC}$, resection, transplantation or local ablation are the first-line recommended therapy. ${ }^{4,12}$ However, in clinical practice, resection, transplantation or local ablation is infeasible in many cases. ${ }^{10}$ For hepatic resection, hepatic reservoir function and future liver volume or remnant liver volume after resection should be considered in order to prevent postoperative hepatic insufficiency.' Thus, hepatic resection cannot be considered for HCC patients with limited hepatic reservoir function or remnant liver volume. For ablation, many HCC patients cannot undergo radiofrequency ablation due to inability to visualize the tumor. ${ }^{13}$ Even if visible, the risk of thermal injury and heat sink effect limit its applicability. ${ }^{1}$ For them, TACE is a valuable and sometimes the best option. It is an important clinical issue to develop and apply methods that can improve treatment outcome for very 

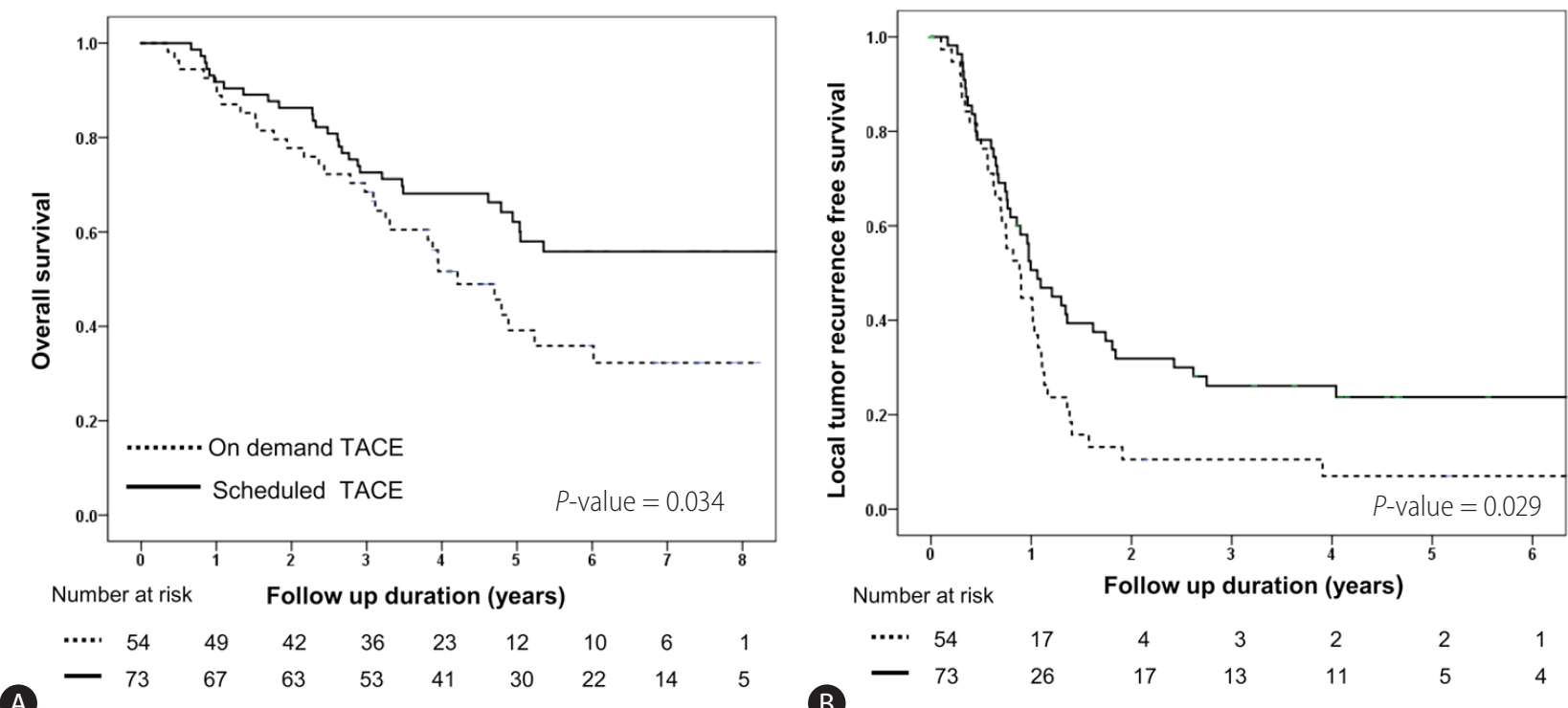

(4) Number at risk Follow up duration (years)

$\begin{array}{cccccccccc}\cdots \cdots & 54 & 49 & 42 & 36 & 23 & 12 & 10 & 6 & 1 \\ \text { A } & 73 & 67 & 63 & 53 & 41 & 30 & 22 & 14 & 5\end{array}$

B

Figure 3. Comparison of overall survival and local tumor recurrence-free survival in scheduled second TACE and on-demand groups of BCLC A stage patients ( $n=127)$. Kaplan-Meier curves showing the overall survival rate $(A)$ and local tumor recurrence-free survival (B) in the scheduled second TACE $(n=73)$ and on-demand groups $(n=54)$. There were significant differences in overall survival $(39.1 \%$ vs. $62.1 \%$ at 5 years, $P=0.034)$ and local tumor recurrence-free survival ( $44.7 \%$ vs. 50.6\% at 1 year, $P=0.029)$ between the second TACE and on-demand groups. TACE, transarterial chemoembolization; BCLC, Barcelona clinic liver cancer.

early or early-stage HCC patients treated with TACE.

In this study, we found that scheduled second TACE was an independent factor associated with overall survival, along with $\mathrm{BCLC}$ stage and underlying liver disease. To the extent that we are able to understand based on what is know, this is the first report that shows improved survival by scheduled second TACE in this subgroup. It has clinical implication as performing scheduled second TACE is a modifiable risk factor. Although the exact reason why scheduled second TACE benefited patients' survival remains to be determined, several mechanisms suggest that our observation can be plausible. It is well known that radiological complete remission (no viable tumor) after TACE does not always match histological complete tumor necrosis. ${ }^{8,9}$ Lipiodol artifact can lead to an underestimation of residual viable tumor when response is evaluated by CT. ${ }^{14-16}$ For post-TACE CT assessment, dense lipiodol uptake has been proven to be a poorly specific marker of a complete histological response. ${ }^{9}$ Scheduled second TACE may further enhance tumor necrosis, which may result in better local tumor recurrence free survival and better overall survival than the ondemand approach.

In this study, BCLC stage in those who received scheduled second TACE were more advanced than those with the on-demand approach. As BCLC stage was an independent risk factor for overall survival, recurrence free survival, and local recurrence free sur- vival, we underwent subgroup analysis stratified by BCLC stage. we found more of a benefit of scheduled second TACE on survival of BCLC stage A patients. However, such benefit was less obvious in BCLC stage 0 patients. BCLC $0 \mathrm{HCC}$ is composed of single tumor, while those with BCLC A include multiple tumors (two or three nodules; 61 patients had multiple tumors in this study). Golfieri et al. ${ }^{9}$ reported higher degree of tumor necrosis for single nodules than multiple nodules, when treated with TACE. Kinugasa et al. ${ }^{17}$ reported that multiplicity of tumors is a risk factor for tumor recurrence in patients with early-stage HCC who undergone TACE.

However, we should acknowledge that the present study has several limitations. This study is a retrospective study with inherent limitations including potential selection, measurement and misclassification biases. Because of the long study duration, many radiologists were involved in reading $\mathrm{CT}$ findings. There can be inter-observer or intra-observer variation in reading the CT findings between different radiologists. However, as radiologists were unaware of the study aims, measurement errors in reading these $\mathrm{CT}$ findings were independent and non-differential. Kloeckner et al. ${ }^{14}$ suggested that MRI should be used over CT, as MRI is superior to CT for detection of viable tumor residuals after lipiodolbased TACE. Response rate after the first TACE or recurrence rate during follow-up can be varied according to the evaluation meth- 
od. In this study, we included only patients who showed complete response by mRECIST criteria at 1-month follow-up CT evaluation after the first TACE. However, there was no pre-defined protocol for using CT or MRI during follow-up in those patients. The choice of performing scheduled second TACE was made by respective physician without any pre-defined clinical criteria. Those who received scheduled second TACE were composed of patients with more advanced HCC stage, indicating that physicians may have considered several factors in deciding scheduled second TACE. Because of the retrospective nature of this study, we were unable to document exact reason for repeating or not-repeating scheduled second TACE. Therefore, prospective validation studies are needed to confirm our findings.

Despite these limitations, this study provides important clinical information and raises question on TACE strategy. Generally, when performing TACE, treatment response evaluation by mRECIST criteria is recommended. ${ }^{1,4,5}$ However, our data suggest that clinicians should be aware of the potential limitation of response assessment with CT after lipiodol based TACE. It might be better to suspect incomplete tumor necrosis in case of BCLC A tumor when treated with TACE for the first time. For them, scheduled second TACE strategy can be a valuable option to further improve clinical outcome. These data call for well-controlled trials that can adequately answer whether scheduled second TACE in the absence of radiological evidence is a better approach than on-demand approach. Still, a significant proportion of early stage HCC patients are initially treated with $\mathrm{TACE}^{6}{ }^{6}$ this issue warrants further validation.

\section{Financial support}

This paper was supported by Research \& Business foundation of Sungkyunkwan University (Grant number: S-2015-0047-000).

\section{Author's contributions}

Study design: Choi MS; Statistical analysis: Kim JH, Sinn DH; Writing of the draft manuscript: Kim JH, Sinn DH, Choi MS; Data collection: Kim JH, Kang W, Paik YH, Choi MS, Lee JH, Koh KC, Paik SW; Critical revision of the manuscript: Kang W, Paik YH, Choi MS, Lee JH, Koh KC, Paik SW. All authors approved the final submission.

\section{Conflicts of Interest}

The authors have no conflicts to disclose.

\section{SUPPLEMENTARY MATERIALS}

Supplementary materials are available at Clinical and Molecular Hepatology website (http://www.e-cmh.org).

\section{REFERENCES}

1. Korean Liver Cancer Study Group (KLCSG); National Cancer Center Korea (NCC). 2014 KLCSG-NCC Korea practice guideline for the management of hepatocellular carcinoma. Gut Liver 2015;9:267-317.

2. Llovet JM, Real MI, Montaña X, Planas R, Coll S, Aponte J, et al. Arterial embolisation or chemoembolisation versus symptomatic treatment in patients with unresectable hepatocellular carcinoma: a randomised controlled trial. Lancet 2002;359:1734-1739.

3. Lo CM, Ngan H, Tso WK, Liu CL, Lam CM, Poon RT, et al. Randomized controlled trial of transarterial lipiodol chemoembolization for unresectable hepatocellular carcinoma. Hepatology 2002;35:1164-1171.

4. Bruix J, Sherman M; American Association for the Study of Liver Diseases. Management of hepatocellular carcinoma: an update. Hepatology 2011;53:1020-1022.

5. European Association For The Study Of The Liver, European Organisation For Research And Treatment Of Cancer. EASL-EORTC clinical practice guidelines: management of hepatocellular carcinoma. J Hepatol 2012;56:908-943.

6. Park JW, Chen M, Colombo M, Roberts LR, Schwartz M, Chen PJ, et al. Global patterns of hepatocellular carcinoma management from diagnosis to death: the BRIDGE Study. Liver Int 2015;35:2155-2166.

7. Lencioni R, Llovet JM. Modified RECIST (mRECIST) assessment for hepatocellular carcinoma. Semin Liver Dis 2010;30:52-60.

8. Choi BI, Kim HC, Han JK, Park JH, Kim YI, Kim ST, et al. Therapeutic effect of transcatheter oily chemoembolization therapy for encapsulated nodular hepatocellular carcinoma: CT and pathologic findings. Radiology 1992;182:709-713.

9. Golfieri R, Cappelli A, Cucchetti A, Piscaglia F, Carpenzano M, Peri $E$, et al. Efficacy of selective transarterial chemoembolization in inducing tumor necrosis in small $(<5 \mathrm{~cm})$ hepatocellular carcinomas. Hepatology 2011;53:1580-1589.

10. Kim KM, Sinn DH, Jung SH, Gwak GY, Paik YH, Choi MS, et al. The recommended treatment algorithms of the $B C L C$ and $H K L C$ staging systems: does following these always improve survival rates for HCC patients? Liver Int 2016;36:1490-1497.

11. Korean Liver Cancer Study Group, National Cancer Center Korea. Practice guidelines for management of hepatocellular carcinoma 2009. Korean J Hepatol 2009;15:391-423.

12. Yu SJ. A concise review of updated guidelines regarding the management of hepatocellular carcinoma around the world: 2010-2016. Clin Mol Hepatol 2016;22:7-17. 
13. Choi HJ, Ko SY, Choe WH, Seo YS, Kim JH, Byun KS, et al. Clinical features of acute viral hepatitis B in Korea: a multi-center study. Korean J Hepatol 2011;17:307-312.

14. Kloeckner R, Otto G, Biesterfeld S, Oberholzer K, Dueber C, Pitton $M B . M D C T$ versus MRI assessment of tumor response after transarterial chemoembolization for the treatment of hepatocellular carcinoma. Cardiovasc Intervent Radiol 2010;33:532-540.

15. Lee JK, Chung YH, Song BC, Shin JW, Choi WB, Yang SH, et al. Recurrences of hepatocellular carcinoma following initial remission by transcatheter arterial chemoembolization. J Gastroenterol Hepatol 2002;17:52-58.

16. Chung YH. A strategy for early detection of recurrent hepatocellular carcinoma following initial remission by transcatheter arterial chemoembolization. Intervirology 2005;48:46-51.

17. Kinugasa H, Nouso K, Takeuchi Y, Yasunaka T, Onishi H, Nakamura $\mathrm{S}$, et al. Risk factors for recurrence after transarterial chemoembolization for early-stage hepatocellular carcinoma. J Gastroenterol 2012;47:421-426. 